AUTORES:

Diego H. Figueiredo

Diogo H. Figueiredo ${ }^{1}$

Marcelo de Oliveira Matta ${ }^{1}$

Faculdade de Educação Físic Juiz de Fora Minas Gerais, Brasil.

\section{Alterações da força explosiva após o período preparatório \\ em jovens atletas de Futebol}

\section{PALAVRAS CHAVE:}

Futebol. Força explosiva. Salto vertical.

2. Amadeu. S. (2008). Educar para a era digital. Revis-

ta Educação: os intelectuais que renovam a pedagogia.

São Paulo: Segmento, n. 108, p. 34

3. Marquès, P. (2000). Las TIC y sus aportaciones a

la sociedad. Disponivel em: <http://www.pangea.org/ peremarques/tic.htm>. Acesso em: 16 mai. 2013.

4. Sebriam, D. C. (2009). Utilização das tecnologias

da informação e comunicação no ensino de Educação Física. (Dissertação de Mestrado). Programa Erasmus Mundus - Mestrado em Engenharia de Mídias para a Educação - Portugal, Espanha e França

5. Bzuneck, J. A. (2000). As crenças de auto-eficácia dos professores. In: F.F. Sisto, G. de Oliveira, \& L. D. T. Fini (Orgs.). Leituras de psicologia para formação de professores. Petrópolis, Rio de Janeiro: Vozes, 2000 6. Barbosa, M. O. ; Melo, F. A. P. ; Lima, A. S. ; Santos, F. M. ; Montenegro, P. C. A. (2011). O PONTO DE VISTA DOS PROFESSORES DE EDUCAÇÃO FÍSICA SOBRE P PIBID EM SEUS CONTEXTOS ESCOLARES. In: XVII Congresso brasileiro de ciências do esporte, Porto Alegre. Ciência e compromisso social. Porto Alegre: UFRGS. v. XVII. p. 1-9

7. Silva, A. M.; Penha, M. G. (2012). 0 uso das tecnologias no ensino fundamental: novos desafios para o professor de educação física em uma escola pública de Goiânia Revista Eletrônica de Pedagogia. Jataí: V. 2 nำ13.

8. Nascimento, R. J. (2003). Uso das tecnologias de informação e comunicação na formação de professores de educação física e desporto: estudo experimental num módulo de basquetebol. (Tese de Doutorado). Lisboa, Portugal. Faculdade de Motricidade Humana, Universidade Técnica de Lisboa.

9. Barbosa, C. L. (1997). Educação Física Escolar da alienação à libertação. 1르 ed. Petrópolis: Vozes.

11. Gil, A. C. (2008). Métodos e técnicas de pesquisa cial. 60 ed. São Paulo: Atlas.

2. Machado, J.; Tijiboy, (2005). A. Redes sociais viruais: um espaço para efetivação da aprendizagen coperativa. 11. Novas tecnologias na educação, V. 3 № 1, Maio, 2005. Disponível em: <http://www.inf.ufes. br/ロcvnascimento/artigos/a37_redessociaisvirtuais. df> Acesso em: 24 mai. 2013

13. Andrade, A. (2011). 0 uso das Tecnologias na Educação: computador e internet. (monografia) Consórcio setentrional de EAD, Universidade de Brasília/Universidade estadual de Goiás no curso de licenciatura em biologia à distância.

14. IBGE. (2013). Acesso à internet no Brasil. Disponível em: <http://diariodonordeste.globo.com/noticia asp?codigo=359284>. Acesso em: 17 maio

\title{
RESUMO
}

0 processo de treinamento no futebol, assim como em vários esportes é extremamente complexo, pois trata-se de uma modalidade onde os atletas realizam funções específicas dentro da partida. Em sua grande maioria, os momentos decisivos do jogo ocorrem em lances de alta intensidade, com curtas distâncias e durações, sendo evidente a solicitação predominante dos membros inferiores. Nesta perspectiva, as manifestações de força estão ganhando importância como indicador de desempenho físico, e a técnica de salto vertical vem sendo utilizado como principal método para a medida de força. 0 principal objetivo deste trabalho foi refletir sobre questões relacionadas ao desenvolvimento da força explosiva em jovens futebolistas, durante um período de quatro semanas de treinamento preparatório. Participaram do estudo 17 atletas do sexo masculino, divididos pela categoria infantil $(n=9)$ e juvenil $(n=8)$, submetidos a avaliação de força explosiva de membro inferiores no inicio e ao final de uma pré-temporada. Os resultados sugerem que houve um aumento significativo no desempenho da força explosiva de membros inferiores entre o início e o final do período preparatório.

Correspondência: Diego Hilgemberg Figueiredo. Faculdade de Educacão Física e Desportos (Faefí) da Universidade Federal de Juiz de Fora, Minas Gerais, Brasil (diegohilgemberg@ @otmail.com). 


\section{Changes in explosive strength}

\section{after the preparatory period}

\section{in young soccer players}

\section{ABSTRACT}

The training process in football, as well in several sports, is extremely complex, once is a type of sport in which the athletes execute specific functions within the game. For the most part, the decisive moments of the game occurs in high intensity plays, with short distances and duration, being evident the predominant demand of the lower limbs. In this sense, the strength characteristics are gaining importance as indicators of physical performance, and, at the same time, the vertical jump technic is starting to be use as the most important method to measure strength. The goal of our study is to analyze the issues related to the explosive strength in young football players, during a period of four weeks in preparatory training. Our sample was composed by 17 male athletes, divides in two categories: 14-15 years old; and 16-17 years old. We analyzed the explosive strength as the lower limbs in the beginning and at the end of the preparatory training period. Our results suggest that there were a significant increase in this variable.

\section{KEY WORDS}

Football. Explosive strength. Vertical jump.

\section{INTRODUÇÃo}

O processo de treinamento de futebol, assim como em vários esportes coletivos é extremamente complexo, principalmente ao se tratar de uma modalidade em que os atletas desempenham funções específicas dentro da partida. Pelo caráter intermitente das atividades motoras, em desportos como futebol, o desempenho está associado com a habilidade que $o$ atleta possui em realizar esforços intensos ${ }^{22}$.

Apesar de o futebol apresentar características de uma atividade de longa duração, os momentos decisivos do jogo, em sua maioria, ocorrem em lances de alta intensidade, com curtas distâncias e durações, fazendo apelo a movimentos de tipo explosivo. Rebelo ${ }^{27}$ afirma que no jogo as ações consideradas importantes estão ligadas a uma produção elevada de potência a nível muscular

Portanto a necessidade de desencadear esforços curtos e intensos é um fator determinante para os futebolistas, sendo evidente a solicitação predominante dos membros inferiores.

Podemos afirmar que as manifestações de força estão ganhando, cada vez mais, importância como uma variável no desempenho físico dos atletas de futebol. Nesta perspectiva, o salto vertical tem sido destacado na literatura como principal método de medida de força explosiva de membros inferiores para desportistas, sendo considerado um meio de preparação essencial para o aprimoramento da força e velocidade ${ }^{8,14,16}$.

Neste estudo, procurou-se refletir sobre questões relacionadas ao desenvolvimento da força explosiva em jovens futebolistas, durante um período de quatro semanas de treinamento preparatório.

\section{DEFINIÇÃO DE FORÇA}

Força muscular é entendida como a capacidade motora que permite ao homem vencer uma resistência ou opor-se a um esforço intenso, através da sua musculatura ${ }^{23}$.

Já no âmbito desportivo González-Badillo e Ayestarán ${ }^{17}$ referem que força é a capacidade do músculo produzir tensão ou contrair-se.

A produção de força está associada à capacidade de se gerar impulso. A magnitude de força está associada à função de três fatores: a coordenação intermuscular, coordenação intramuscular e a força com a qual o músculo reage a um impulso nervoso ${ }^{28}$. 
TIPOS DE MANIFESTAÇÃO DE FORÇA

Ao nos referirmos à força, devemos primeiramente identificar qual a componente que pretendemos trabalhar, pios ela pode se manifestar sobre várias formas ${ }^{24}$. González-Badillo e Ayestarán ${ }^{17}$ sustentam esta ideia quando afirmam que no homem a força nunca se manifesta de forma pura.

A força em suas diversas manifestações pode ser dividida conforme a análise que se faz e a partir de critérios de classificação, a análise pode ser feita em relação:

- Quantidade de massa muscular (Força Geral e Força Local)

- Especificidade da modalidade (Força Geral e Força Específica)

- Tipo de trabalho muscular (Força Dinâmica e Força Estática)

- Peso Corporal (Força Absoluta e Força Relativa)

- Características dos exercícios de força (Força Máxima, Força Rápida e Força Resistente).

Porém existe uma diferenciação proposta por Vittori ${ }^{32}$, citado por Correia ${ }^{13}$, onde se divide as diferentes manifestações de força em uma nova tipologia que engloba a maior parte das relações descritas anteriormente em: Manifestação ativa de força, Manifestação reativa de força e Manifestação estática de força.

FORÇA ATIVA

A manifestação ativa de força é entendida pelo efeito da força produzida por um ciclo simples de trabalho muscular, aquele de encurtamento da parte contrátil ${ }^{4,6}$. É a tensão gerada pela ação de uma contração muscular voluntária.

Em função de sua magnitude, velocidade de execução e tempo de duração é possível encontrar diferentes manifestações ativas de força, sendo conhecidas duas expressões, a força máxima e a força explosiva. Como o objeto do presente estudo está voltado para a força explosiva vamos nos atentar somente a ela.

Força explosiva é aquela força que vem expressa por uma ação de contração mais rápida possível, para transferir à sobrecarga a ser vencida na maior velocidade possível de contração muscular. Segundo Schmidtbleicher ${ }^{29}$ trata-se da capacidade que o sistema muscular tem de produzir o maior impulso no menor período de tempo.

\section{FORÇA REATIVA}

A manifestação de força reativa é compreendida pelo efeito da força produzida por um ciclo duplo de trabalho muscular. Comumente os segmentos corporais estão sujeitos a forças de impacto, como nos saltos e corridas ou ainda pelo efeito de forças externas como a gravidade que acabam envolvendo a realização de ciclos musculares de alongamento-encurtamento (CAE), sendo um mecanismo fisiológico que tem como função elevar a eficiência mecânica do movimento ${ }^{21}$. Na realização de um CAE uma grande quantidade de energia elástica é armazenada durante a fase de alongamento muscular devido principalmente a um estado de pré-ativação dos músculos antagonistas. Posteriormente esta energia elástica é liberada na fase de encurtamento (fase concêntrica) subsequente na forma de energia cinética, que se traduzirá em um aumento da força e em uma baixa ativação nervosa, no sentido de melhorar o desempenho em situações de esforço máximo e submáximo.

Alguns estudos tem demostrado que a duração do tempo de contato com o solo permite distinguir dois tipos de CAE assim como dois tipos de manifestação reativa: a Força Elástica-Explosiva e a Força-Explosiva-Reativa ${ }^{17}, 29$. Iremos focar nossa atenção somente a primeira.

A Força Elástica-Explosiva é caracterizada pela realização de CAE longos, caracterizados por amplos movimentos angulares ${ }^{9}$. Para Correia ${ }^{13}$, a fase excêntrica do movimento não se realiza em uma velocidade elevada devido ao acentuado deslocamento angular das articulações envolvidas.

\section{MATERIAL E MÉTODOS}

Participaram deste estudo 17 atletas do sexo masculino, sendo nove pertencentes à categoria infantil (Sub-15) com idade média de $14,33 \pm 0,71$ anos e oito pertencentes à categoria juvenil (Sub-17) com idade média de $16 \pm 0,71$ anos, integrantes da equipe de futebol do Sport Clube Juíz de Fora, da cidade de Juíz de Fora (Minas Gerais), que se encontravam em pré-temporada. $\mathrm{O}$ presente estudo faz parte do projeto submetido e aprovado pelo CEP-HU CAS/UFJF, parecer $N^{\circ} 009 / 11$

Para atingirmos nossos objetivos iremos utilizar, neste estudo, a Variável Física de Força Explosiva, o Teste de Força Explosiva para o Componente Contrátil (SJ) e para a Componente Elástica (CMJ), que passaremos a descrever.

As variáveis de estudo relacionadas ao desempenho físico de força explosiva correspondem à força explosiva (FE) e força explosiva elástica (FEE). Para determinar os valores de FE e FEE em membros inferiores foram utilizados os resultados obtidos nos testes de Squat Jump (SJ) e Counter Moviment Jump (CMJ) respectivamente, proposto por Bosco ${ }^{5}$. Para análise dos saltos, foi utilizada plataforma de contato CEFISE $^{\circledR}$, conectada ao sistema para a medida de salto Jump System ${ }^{\circledast}$.

Para análise de Força Explosiva em seu Componente Contrátil (SJ) o atleta assumia a posição estática de flexão dos joelhos a $90^{\circ}$, mãos na cintura, evitando assim qualquer influência do movimento dos braços sobre os saltos, não era permitido ao atleta um novo declínio de seu centro de gravidade (CG), sendo o movimento realizado somente na direção ascendente. Realizado tal procedimento, a energia potencial elástica acumulada era perdida na forma de calor, devido a posição estática do atleta, sendo o salto realizado somente com a capacidade que os grupos musculares apresentam de gerar força sem a utilização do $C A E^{18}$. 


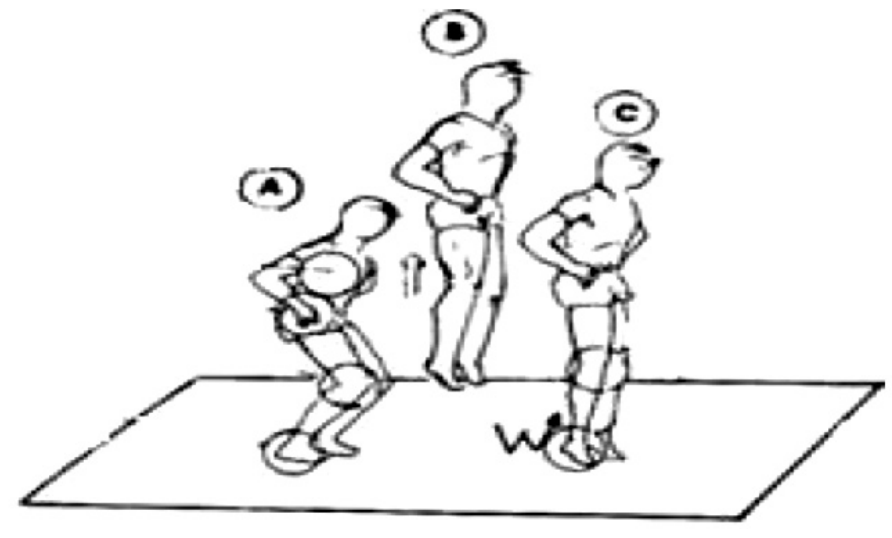

FIGURA 1 - Representação esquemática do Squat Jump (BOSCO, 1999)

Para a Componente Elástica (CMJ) foi utilizada a técnica de salto com contra movimento, em que era permitido ao atleta realizar a fase excêntrica e concêntrica do movimento e assim como na técnica anteriormente descrita realizado com as mãos sobre o quadril. A transição da fase descendente para a ascendente deveria ser realizada o mais rápido possível, desta forma o CAE poderia ser utilizado, o que produziria uma maior geração de força e uma maior elevação do centro de gravidade.

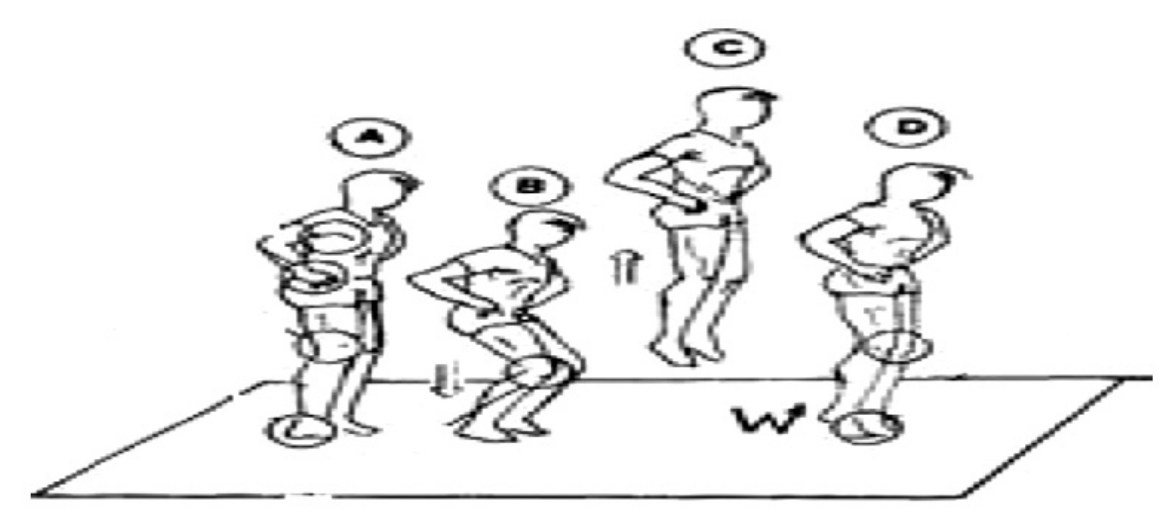

FIGURA 2 - Representação esquemática do Counter Moviment Jump (BOSCO, 1999) s.
Os testes foram realizados em dois momentos distintos, no início e no final do período preparatório de quatro semanas. Os testes de saltos verticais foram empregados na seguinte ordem: SJ e CMJ, sendo precedidos por um aquecimento específico, com duração aproximada de 10 minutos, direcionada para a ativação neuromuscular aos testes de saltos verticais. Cada indivíduo executou três tentativas, o descanso entre as tentativas foi de pelo menos um minuto, o melhor resultado das tentativas foi avaliado como resultado dos testes.

Para descrição dos dados recorreu-se aos procedimentos habituais da estatística descritiva, média e desvio padrão. Para a associação entre os dados de força explosiva, entre o início e o final da pré-temporada, foi utilizado o teste estatístico teste $t$ de Student, e o delta percentual foi empregado para demostrar a magnitude das diferenças, sendo o nível de significância estabelecido para $p \leq 0,05$.

\section{RESULTADOS}

Na observação dos dados pôde-se constatar que houve um aumento nos desempenhos da força explosiva e da força explosiva elástica para a categoria sub-15, entre o início e o final do período preparatório.

\begin{tabular}{|c|c|c|c|c|}
\hline & $\begin{array}{c}\text { alteracōes da } f \\
\text { em futeb }\end{array}$ & $\begin{array}{l}\text { A } 1 \\
\text { sira após quat } \\
\text { categoria sub- }\end{array}$ & manas de prep & \\
\hline \multirow{2}{*}{ Variáreis } & Antes do PP & Após o PP & $\Delta \%$ & \multirow{2}{*}{$\mathbf{p}$} \\
\hline & Média \pm DP & Média $\pm \mathrm{DP}$ & Média $\pm D P$ & \\
\hline $\mathrm{FE}(\mathrm{cm})$ & $26,2 \pm 1,5$ & $28,3 \pm 1,6$ & $8,3 \pm 4,0$ & $<0,05^{\circ}$ \\
\hline FFE (cm) & $28,2 \pm 1,2$ & $31,3 \pm 1,6$ & $11,1 \pm 5,0$ & $<0,05^{\circ}$ \\
\hline
\end{tabular}

Para a categoria Sub-17 podemos perceber que houve aumento nos desempenhos da força explosiva e da força explosiva elástica entre o início e o final do período preparatório. 


\begin{tabular}{|c|c|c|c|c|}
\hline \multirow{3}{*}{ Variáreis } & \multicolumn{4}{|c|}{$\begin{array}{c}\text { TABELA 2 } \\
\text { Descritivo da alteracóoes da força explosiva após quatro semanas de preparação } \\
\text { em futebolistas da categoria sub- } 17\end{array}$} \\
\hline & Antes do PP & Após o PP & $\Delta \%$ & \multirow{2}{*}{$\mathrm{p}$} \\
\hline & Média \pm DP & Média \pm DP & Média $\pm D P$ & \\
\hline $\mathrm{FE}(\mathrm{cm})$ & $30,4 \pm 2,3$ & $33,5 \pm 2,2$ & $10,1 \pm 4,0$ & $<0,05^{\circ}$ \\
\hline FFE (cm) & $34,7 \pm 2,5$ & $37,8 \pm 2,4$ & $9,1 \pm 3,3$ & $<0,05^{*}$ \\
\hline
\end{tabular}

\section{DISCUSSÃo}

É possível observar que após quatro semanas de período preparatório houve um comportamento de evolução nas variáveis de força, os quais comprovaram que os desenvolvimentos de força explosiva pelos componentes contráteis e elásticos apresentaram aumento.

Ao efetuarmos uma comparação entre os valores obtidos no $\mathrm{CMJ}$ com os verificados no $\mathrm{SJ}$, constatamos em termos gerais, como seria de se esperar, que os resultados de força explosiva foram maiores no teste de CMJ em relação aos resultados do SJ. Este fato parece estar associado ao pré-alongamento muscular realizado, o que aumenta a produção de força durante a contração concêntrica imediata ${ }^{7,30}$. Ao averiguar as diferenças percentuais entre a altura da elevação do centro de gravidade, avaliada pela altura do salto em CMJ e SJ, expresso pelo índice de elasticidade, encontramos valores na ordem de 10\% (Sub-15 $10,4 \%$ e Sub-17 13,1\%).

Segundo Baker ${ }^{1}$, a melhoria do desempenho no $\mathrm{CMJ}$, comparativamente ao $\mathrm{SJ}$, situa-se na ordem de 10 a $20 \%$, para o sexo masculino, sendo que valores abaixo do mínimo significaria uma má utilização do CAE. Harman, Rosenstein, Frykman e Rosenstein ${ }^{19}$ encontraram uma diferença de $12 \%$ na altura de elevação do $C G$, do $C M J$ para o SJ,

A obtenção de tais índices de elasticidade sugere uma maior e melhor utilização da energia potencial elástica pelos indivíduos do presente estudo, devido, provavelmente, a uma melhor coordenação de movimento, ao nível das fases excêntrica e concêntrica dos membros inferiores ao longo do processo de treino.

Já o aumento de desempenho da FE, possivelmente, está relacionado ao componente neural, dentre os quais são sugeridas modificações no recrutamento das unidades motoras ${ }^{31}$. Para alguns autores as estruturas neurais entendidas como o recrutamento seletivo de um maior número de unidades motoras, ativadas a uma maior frequência e de maneira melhor sincronizada, facilitam a contração muscular causando assim uma melhora substancial no salto vertical ${ }^{11,20}$. Alguns estudos têm demostrado aumentos significativos no desempenho da FE e FEE com preparações entre 8 a 12 semanas em futebolistas ${ }^{12,33}$.

0 potencial elástico do músculo esquelético é uma propriedade que pode ser aprimorada através do treino ${ }^{2}$, portanto o aumento do número de anos de treinamento poderá levar a uma melhoria do aproveitamento, por parte dos futebolistas da energia potencial elástica e da capacidade contrátil do músculo.

Nunes26 avaliando o desempenho da FE e FEE no período pré-competitivo em uma equipe da primeira divisão do Campeonato Brasileiro, demostrou valores médios de $36,22 \pm 3,39 \mathrm{~cm}$ e 41,05 $\pm 3,57 \mathrm{~cm}$. Já Casajús ${ }^{10} \mathrm{em}$ um estudo com futebolistas pertencentes a uma equipe da primeira divisão da Espanha também em período pré-competitivo, apresenta valores médios de $39 \mathrm{~cm}$ e $41,4 \mathrm{~cm}$ para FE e FEE. Podemos perceber que os participantes do presente estudo apresentam valores médios abaixo do encontrado nos estudos supracitados.

Wong, Chamari, Wisloff ${ }^{33}$ ao investigarem o efeito do treinamento no desempenho da força explosiva após um período de preparação, encontram diferenças significantes $(p<0,05)$ após a aplicação de treinamento de força, quando comparado ao grupo controle. Com base nos dados apresentados podemos indicar que existe a treinabilidade, tanto para força ativa ( $\mathrm{FE})$, como também na força reativa ( $\mathrm{FEE})$.

Vários meios de treinamento têm sido comumente utilizados e desenvolvidos na tentativa de maximizar o salto vertical, principalmente aqueles que possam aumentar a quantidade de energia armazenada e reutilizada pelo músculo durante o CAE. Dentre os exercícios que exploram este ciclo podemos destacar os chamados exercícios pliométricos, definidos como aqueles que "ativam o ciclo excêntrico-concêntrico do músculo esquelético provocando sua potenciação elástica, mecânica e reflexa" ${ }^{25}$.

Diallo, Dore, Duch e Van Praagh ${ }^{15}$ constataram aumentos no desempenho em ambos os testes de salto verticais $\mathrm{SJ}(\mathrm{p}<0,05), \mathrm{CMJ}(\mathrm{p}<0,01)$ em um grupo submetido a um programa de treinamento que englobava exercícios pliométricos em comparação ao grupo controle, demostrando a existência de aumentos de desempenho da força em programas de treinamento específicos.

Outro fator condicionante para a evolução dos níveis de força que podemos considerar é a função idade. A função explosiva muscular atinge seu ponto mais alto entre os 20-25 anos, decrescendo com a idade de uma forma quase linear ${ }^{3}$.

os resultados obtidos no presente estudo sugerem que o treinamento intensivo combinado com estímulos específicos do treinamento dos futebolistas tem proporcionado qualidades físicas específicas no desempenho das expressões de força. 
Os resultados sugerem que houve um aumento significativo no desempenho da Força Explosiva (FE) e da Força Explosiva Elástica, entre o início e o final do período preparatório, sendo encontrados valores superiores em ambas variáveis para a categoria Sub-17 quando comparada a categoria Sub-15.

Resultados como os encontrados no presente estudo são de grande importância para a prescrição de treinamentos de caráter específico de saltos, devido sua importância no rendimento dos jogadores e também na identificação de possíveis problemas nas variáveis que compõem o salto vertical.
1. Baker D (1996). Improving, vertical jumping performance through genera, special, and specific strength training: a brief review. J Strength Cond Res10:131-136. 2. Bosco C (1981). New tests for the measurement of anaerobic capacity in jumping and leg extensor muscle activity. IVBF Official Magazine 1: 37-42

3. Bosco C (1982). Stretch-shortening cycle in skeletal muscle function; with special reference to elastic energy and potentiation of myoelectrial activity. Studies in Sport, Physical Education and Health.

4. Bosco C (1998). L'effetto della vibrazione sulla forza musculare e sul profile ormonale in atleti. Atletic Studi 4:7-14

5. Bosco C (1999). Strength assessment with the Bosco's test. Italian Society of sport Science 6. Bosco C (2007). A força muscular: Aspectos fisiológicos e aplicações práticas. São Paulo: Phorte

. Bosco T (2000). Lafuerza Muscular. Aspectos metodológicos. Editorial Inde

Brown LE, Weir JP (2001). (ASEP) Procedures Recommendation I: Accurate Assessment of Muscular Strength And Power. J Exe Phy 4: 1-21

Carvalho C, Carvalho A (1996). A Força em Crianças e Jovens. 0 seu desenvolvimento e treinabilidade. Lisboa: Livros Horizonte

10. Casajús JA (2001). Seasonal variation in fitnes variables in professional soccer players. J Sports Med Phys Fitness 41: 463-469

11. Cavagna GA, Dusman B, Margaria R (1968). Posittve work done by a previously stretched muscle. J App Physiol 24: 21-32

Chelly MS, Ghenem MA, Abid K, Hermassi S, Tabak Z, shepard RJ (2010). Effects of in-season short-term plyometric training program on leg jump- and sprin performance of soccer players. $J$ Strength Cond Res 24: 6783-79

13. Correia R (2003). A força no futebol. A importância da força básica (musculação) em acções motoras específicas do futebol: saltos e sprints. Dissertação de Mestrado. FCDEF-UP. Porto

14. Cronin JB, Hansen KT (2004). Strength and power pre dictors os sports speed. J Strength Cond Res 19: 349-357
15. Diallo O, Dore E, Duche P, Van Praagh E (2001). Effects of plyometric training followed by a reduced training program me on physical performance in prepubescent soccer players. J Sports Med Phys Fitness 41: 342-48

16. Feltner ME, Bishop EJ, Perez CM (2004). Segmental and Kinetic contributions in vertical jumps performed with and without an arm swing. Res Q Exerc Sport 75: 216-230

17. González-Badillo J, Ayestarán E (2001). Fundamentos do treinamento de força. Aplicações ao alto rendimento desportivo. Editora Artmed 18. Goubel $F(1997)$. Series elastic behavior during the Stech-Shortening cycle. J Appl Biomech 3: 439-43 19. Harman E, Rosenstein MT, Fraykman PN, Rosenstein RM (1990). The effects of arms and countermovement on vertical jumping. Med Sci Sports Exerc 22: 826-33

20. Komi PV (1986). The stretch-shortening cycle and human power output. In: Jones L, McCartney N, McCo-

nas A (ed). Human Kinetics Champaign. Tlinois, 27-42

21. Komi PV (1992). Strength and Power in sport. London: Blackwell Scientific Publicatio

22. Krustrup P, Mohr M, Amstrup T, Rysgaard T, Johansen J, Strensberg A, Pedersen PK, Bangsboo (2003). The Yo-Yo Intermitent recovery Test: pshysiological response, reability and validity. Med Sci Sports Exerc 35: 697-705

23. Manno R (1992). Les Bases de l'entrainement sportif. Editions Renne

Meylan C, Malesta D (2009). Effects of in- season plyometric training within soccer practice on explosive actions of young players. J Strength Cond Res 23: 2605-13 24. Mill-Homens P (1996). Metodologia do treino desportivo. Edições FMH-ULT

25. Moura, NA (1988). Treinamento pliométrico: introdução ás suas bases fisiológicas, metodológicas e 0 efeito do treinamento. Rev. Brasi. Ciência e Movimento 2: 30-40 26. Numes, CG (2004). Associação entre a força explosiva e a velocidade de deslocamento em futebolistas profissionais. Dissertação (Mestrado em Ciências do Desporto) - Faculdade de Educação Física, Unicamp. Campinas 
Vera Lúcia F. P. Fernandes ${ }^{1}$

Marcelo Luís R. S. Tavares ${ }^{1}$

28. Ramsay JA, Blinkei C, Garner S, MacDougall JD,

Sale DG (1990) Strength training effects in prepubes-

cent boys. Med Sci Sports Exerc 22: 605-614

29. Schmitbleicher D (1992). Training for Power

Events. In: Komi PV. London: Blackwell Scientific Pu-

blication, 381-396

30. Seabra A, Catela D (1998). Maturação, crescimen-

to físico e prática desportiva em crianças. Revista Ho-

rizonte 14: 15-17

31. Van Praagh E, DoréE (2002). Short-term muscle

power during growth and maturation. Sports Med 32:

701-28

32. Vittori (1990). El entrenamiento de la fuerza para

el Sprint. Tomo IV

33. Wong P, Chamari K, Wisloff U (2010). Effects

of 12-week on-field combined strength and power

training on physical performance among $\mathrm{u}-14$ young

players. J Strength Cond Res 24: 644-652.
Ayra Lovisi Oliveira ${ }^{1}$

Ludmila Nunes Mourão ${ }^{1}$

${ }^{1}$ Faculdade de Educação Física

e Desportos, Universidade Federal de Juíz de Fora, Minas Gerais, Brasil.
RESUMO

\section{A produção de feminilidades}

\section{de atletas de Luta Olímpica}

\section{PALAVRAS CHAVE:}

Luta Olímpica. Mulheres. Feminilidade.

Uma feminilidade hegemônica culturalmente construída atribui a passividade, a delicadeza e a beleza como características essenciais das mulheres. As práticas esportivas constituem-se em elementos influenciadores dessa construção social, sendo que as lutas são identificadas como esportes agressivos, de constituição e exercício da masculinidade e, por isso, desaconselhadas às mulheres. Este estudo teve por objetivo analisar as representações de feminilidade de lutadoras profissionais e perceber até que ponto se aproxima e/ ou afastam da feminilidade hegemônica. Baseado no aporte teórico dos estudos de gênero e metodológico das representações sociais percebeu-se que as atletas constroem feminilidades plurais, combinando características culturalmente masculinas e outras da cultura feminina e, dessa forma, deixam suas marcas neste território ainda hegemonicamente masculino das lutas. Faz-se necessário o aprofundamento das pesquisas no tema para que possamos conhecer as representações de outras lutadoras, a fim de contribuir para a ampliação das discussões sobre a pluralidade das formas de ser e viver feminilidades e, assim, dissolver normatizações culturais que ainda buscam dificultar a presença das mulheres no campo das lutas. 